\title{
Sangrado digestivo alto: Una emergencia médica
}

\section{Upper digestive bleeding: A medical emergency}

Pág. 144,152

Recibido: 20-05-2020

Aceptado: 26-06-2020

Ana Sofía Echeverría Flores ${ }^{1}$

William González Villegas ${ }^{2}$

Daniela Polanco Méndez ${ }^{3}$

Joshua Ramírez Garita ${ }^{4}$

Katherine Vargas Aguilar ${ }^{5}$

1. Médico general, Hospital San Rafael de Alajuela, Caja Costarricense del Seguro Social,Costa Rica.

2. Médico general, área de salud de Golfito, Caja Costarricense del Seguro Social,Costa Rica.

3. Médico General, Trabajador Independiente. San José,Costa Rica.

4. Médico general, EBAIS de Cocorí, Caja Costarricense del Seguro Social,Costa Rica.

5. Médico General, Trabajador Independiente. San José,Costa Rica.

\section{RESUMEN}

Elsangradodigestivoaltoesaquelquesepresenta a nivel de esófago, estómago y/o duodeno, se debe principalmente $(80-90 \%)$ a causas no variceales siendo la más frecuente las úlcera péptica, sin embargo también puede ocurrir por la ruptura de várices esofágicas secundarias a hipertensión portal. Entre los factores de riesgo para el desarrollo de esta patología se encuentra la infección por Helicobacter pylori (H.pylori), el uso de antiinflamatorios no esteroideos (AINES), así como el uso de medicamentos antiplaquetarios y anticoagulantes. Se manifiesta como hematemesis y melena principalmente. Representa una emergencia médica, razón por la cual el abordaje inicial consiste en valorar y estabilizar el estado hemodinámico y realizar endoscopia para identificar el origen del sangrado digestivo. El tratamiento definitivo va dirigido a corregir la causa subyacente.

\section{PALABRAS CLAVE:}

Hemorragia, Hematemesis, sangre oculta, várices esofágicas y gástricas, úlcera péptica.

\section{ABSTRACT}

Upper digestive bleeding occurs at the level of the esophagus, stomach and / or duodenum, which is mainly due to non-variceal causes (80$90 \%$ ), being the most common cause peptic ulcers, although it can also be caused by the rupture of esophageal varices secondary to portal hypertension. Among the risk factors 
for the development of this pathology are Helicobacter pylori (H.pylori) infection, the use of non-steroidal anti-inflammatory drugs, as well as the use of antiplatelet and anticoagulant medications. It manifests as hematemesis and melena; and represents a medical emergency, so its initial approach will require evaluation and stabilization of the hemodynamic status and generally endoscopy to identify the cause. Once the cause is found, definitive treatment will be given based on it.

\section{KEY WORDS:}

Hemorrhage, Hematemesis, occult blood, esophageal and gastric varices, peptic ulcer

\section{METODOLOGÍA}

Para la selección de artículos realizada para esta revisión bibliográfica se utilizaron los siguientes criterios de inclusión: publicaciones realizadas entre 2015 y 2020, en idioma inglés y español, relacionadas con las ciencias de la salud, departamentos de medicina interna. Se utilizó la búsqueda en bases de datos como PubMed, MedLine, MedScape, Scielo, Clinical key, The Cochrane Library Plus, UpToDate y Ovid. Utilizando los siguientes criterios de búsqueda "Hemorragia digestiva alta", "varices esofágicas", "Úlcera péptica". Posteriormente se filtraron los de mayor importancia científica lo que nos llevó a un total de 15 artículos a partir de los cuales se recopiló, se revisó y se organizó la información para la redacción de este artículo.

\section{INTRODUCCIÓN}

Sangrado digestivo alto es aquel sangrado que se presenta proximal al ligamento de Treitz, que a su vez forma el ángulo con el mismo nombre, que divide el duodeno del yeyuno (abarcando esófago,estómago y duodeno) (1). Su principal etiología es por causas no variceales, entre las cuales se contempla principalmente la enfermedad por úlcera péptica $2,3,4,5)$. Un pequeño porcentaje es causado por várices esofágicas como consecuencia de la hipertensión portal de diversa etiología (5). Los principales factores de riesgo son el uso de medicamentos antiinflamatorios no esteroideos, aspirina, infección por H.pylori, entre otros $(2,4)$ Las manifestaciones clínicas abarcan desde pacientes asintomáticos, pacientes con síntomas dispépticos y signos de sangrado digestivo alto hasta pacientes que se presentan con hemorragia activa severa, lo cual representa una emergencia médica $(2,5,6)$. El manejo inicial consiste en evaluar y estabilizar el estado hemodinámico del paciente como primer paso (6).

\section{DEFINICIÓN}

Se define como el sangrado que se presenta proximal al ligamento de Treitz. Puede ser originado en esófago, estómago y/o duodeno $(1,4,7)$. Se clasifica según su etiología en no variceal y variceal $(1,3,5,7)$.

\section{EPIDEMIOLOGÍA}

En USA el sangrado digestivo alto afecta aproximadamente 65 personas de cada 100000 cada año $(2,5)$ La incidencia es más alta en el sexo masculino que en el sexo femenino (5) En edad pediátrica la incidencia es de menos del $20 \%$ de todos los casos de sangrado gastrointestinal (7).

Representa una emergencia médica común con una mortalidad de $2-10 \%$ de los pacientes $(4,8)$ Factores de riesgo para la mortalidad incluye la edad avanzada, inestabilidad 
hemodinámica al ingreso hospitalario y presencia de comorbilidades $(2,3)$ Sin embargo, la morbimortalidad ha ido descendiendo a nivel mundial con la erradicación de $\mathrm{H}$. pylori y la prevención en el uso de AINES $(2,3)$.

\section{ETIOLOGÍA}

Las causas no variceales representan un 80 $90 \%$ de los episodios de sangrado digestivo alto, siendo la más frecuente de estas la úlcera péptica $(2,3,4,5,1)$. Entre otras causas no variceales se menciona la esofagitis y el síndrome de Mallory Weiss $(2,3,4,5)$.

La hipertensión portal puede llegar a causar várices esofágicas, estas últimas son una causa de sangrado digestivo alto severo.(5) Pacientes con cirrosis son quienes suelen presentar dichas várices, sin embargo, algunos no padecen de cirrosis $(5,1)$.

-Úlcera péptica

La úlcera péptica es un defecto en la mucosa gástrica o duodenal que atraviesa la mucosa, submucosa y alcanza la capa muscular, originado por la hipersecreción ácida en los individuos que la padecen $(9,10)$ Las manifestaciones clínicas varían desde un cuadro asintomático o leve con síntomas dispépticos hasta cuadros con perforación de la úlcera y sangrado digestivo $(9,10)$ El $70 \%$ de los pacientes que sufren este padecimiento suelen cursar asintomáticos. $(9,10)$ Adultos mayores y pacientes que utilizan AINES son más propensos a cursar asintomáticos, sin embargo, tienden a complicarse más adelante $(1,9,10)$

Los pacientes que sí presentan síntomas manifiestan dolor abdominal como el síntoma más prominente, principalmente dolor epigástrico $(9,10)$ Ocasionalmente el dolor puede localizarse en hipocondrio derecho o izquierdo $(6,9)$ El dolor de la úlcera duodenal se presenta clásicamente de dos a cinco horas después de las comidas ya que se secreta ácido gástrico en ausencia de comida y en las noches entre las $11 \mathrm{pm}$ y las $2 a m$ cuando la secreción de ácido gástrico es máxima.(9) Otros síntomas asociados pueden incluir náuseas, distensión abdominal, sensación de plenitud gástrica ( 9 , 10)

La principal complicación es el sangrado digestivo alto, lo cual se manifiesta con hematemesis o melena.(9) El diagnóstico se realiza a través de la visualización de la úlcera por medio de endoscopia alta $(1,3,10)$

\section{-Esofagitis}

Pacientes que se encuentran hospitalizados por otras razones o que utilizan tubos nasogástricos son en los que se presenta más comúnmente la esofagitis. $(3,5)$ Los pacientes con esofagitis erosiva suelen tener historia de reflujo gastroesofágico $(3,5)$

\section{-Síndrome Mallory-Weiss}

Se trata de una laceración en la mucosa en la unión gastroesofágica causada por antecedente de vómitos, especialmente cuando estos son muy recurrentes o muy intensos $(3,4,11)$ El sangrado por esta causa usualmente es autolimitado $(3,11)$

-Várices esofágicas

Las várices esofágicas son una consecuencia de la hipertensión portal y se presentan en el

\section{(요요요}


$50 \%$ de los pacientes que padecen cirrosis. (5, 12) La presentación clínica puede ser parecida a la de los pacientes con sangrado digestivo alto de causas no variceales (hematemesis, melena, vómito en broza de café).(5) El diagnóstico se realiza por medio de endoscopia $(5,12)$

\section{FACTORES DE RIESGO}

La infección por H. Pylori se considera como uno de los principales factores de riesgo para sufrir sangrado digestivo alto $(2,4)$ El uso de antiinflamatorios no esteroides (AINES) y el uso de medicamentos antiplaquetarios y anticoagulantes también son considerados factores de riesgo el uso concomitante de estos medicamentos aumenta el riesgo al doble $(2,3$, 4).

\section{MANIFESTACIONES CLÍNICAS}

El cuadro clínico del sangrado digestivo alto (SDA) se ve manifestado de manera más común como hematemesis y melena. Otra de las presentaciones que se puede encontrar es la emesis en "broza de café" (vómito conteniendo sangre parcialmente digerida que ofrece este aspecto) $(2,5,6)$.

Algunas condiciones como sangrados bucales, epistaxis, consumo de bebidas/alimentos de color rojo o de Peptobismol pueden presentar síntomas similares a los mencionados anteriormente (2).

La hematemesis se define como vómito con sangre y sugiere sangrado proximal al ligamento de Treitz. La emesis en broza de café sugiere sangrado más limitado $(2,5,6)$.

La hematoquecia es la presencia de sangre roja o marrón en las heces, sin embargo ésta se debe usualmente a sangrado del tracto gastrointestinal bajo, aunque ocasionalmente un sangrado digestivo alto masivo también puede manifestarse con hematoquecia (6).

La melena es la presencia de heces de color negro "alquitranado" y se origina en el $90 \%$ proximal al ligamento de Treitz, también puede originarse por un sangrado en la nasofaringe $u$ orofaringe, intestino delgado o colon derecho (6). Los pacientes asintomáticos pueden presentar como único hallazgo una prueba de sangre oculta positiva (1).

\section{EVALUACIÓN}

Cuando se trata de etiología no variceal inicialmente se valora el estado hemodinámico, mediante una adecuada historia clínica, examen físico y estudios de laboratorio para determinar la severidad del sangrado e identificar la posible causa; además de la resucitación con volumen intravenoso de considerarse necesario $(2,3,8)$; seguido de estudios exploratorios, usualmente endoscopia, que permiten un diagnóstico definitivo y en ocasiones permite la realización de un tratamiento endoscópico (6).

Dentro de las exploraciones iniciales se incluye descartar la presencia de sangre oculta en heces. El paciente con un guayaco positivo que presenta anemia sin deficiencia de hierro debe considerarse la necesidad de complementar con endoscopia tanto baja como alta, según los síntomas asociados (13).

La endoscopia permite detectar importantes lesiones del tracto digestivo alto (13), por ejemplo la úlcera péptica, donde la forma definitiva de establecer el diagnóstico es la visualización directa, con una sensibilidad de alrededor de 90\% (9); sin embargo, entre el 3 al 19\% de los 
sangrados digestivos altos diagnosticados por endoscopia no se logra definir el sitio de sangrado (2). Una vez identificada la lesión, la endoscopia terapéutica puede lograr hemostasia y prevenir sangrados recurrentes en la mayoría de los pacientes (6). Sí se visualiza una úlcera durante la realización de la endoscopia es importante considerar la toma de biopsia (dependiendo de las características de la úlcera). La escala de Forrest permite clasificar la úlcera péptica de acuerdo con la visualización de su estado hemorrágico $(2,6)$. Está indicado tomar biopsia de todas las úlceras con características que sugieran malignidad. La biopsia de rutina no se recomienda en aquellas con características benignas (9). Dicha clasificación agrupa las úlceras en 2 categorías: bajo riesgo (Forrest Ilc y III) y alto riesgo, en las que se recomienda el tratamiento endoscópico (2).

Tabla 1. Escala de Forrest (2)

\begin{tabular}{|c|c|c|}
\hline $\begin{array}{c}\text { Clasificación } \\
\text { de Forrest }\end{array}$ & $\begin{array}{c}\text { Estado } \\
\text { hemorrágico }\end{array}$ & $\begin{array}{c}\text { Porcentaje } \\
\text { de }\end{array}$ \\
\hline la & $\begin{array}{c}\text { Sangrado activo en } \\
\text { chorro }\end{array}$ & $55 \%$ \\
\hline Ib & $\begin{array}{c}\text { Sangrado activo en } \\
\text { puntilleo }\end{array}$ & $55 \%$ \\
\hline Ila & Vaso visible & $43 \%$ \\
\hline IIb & Coágulo adherido & $22 \%$ \\
\hline IIc & $\begin{array}{c}\text { Máculas } \\
\text { planmentadas }\end{array}$ & $10 \%$ \\
\hline III & Base limpia & $5 \%$ \\
\hline
\end{tabular}

La esofagitis se presenta comúnmente en pacientes ya hospitalizados por alguna otra patología y que utilizan sonda nasogástrica, la endoscopia también es importante para el diagnóstico (3).

En cuanto al síndrome de Mallory-Weiss, se suele sospechar la presencia de una laceración de la mucosa por el antecedente de náuseas y vómitos $(3,11)$; la endoscopia permite establecer el diagnóstico y descartar otras etiologías, de igual manera el manejo terapéutico (11). Durante la endoscopia se observa laceraciones en la mucosa de la unión gastroesofágica, con extensiones hacia el cardias del estómago o en dirección al esófago, se describen como rupturas longitudinales de coloración roja de la mucosa y en ocasiones cubiertas por coágulos (11).

\section{MANEJO}

La evaluación inicial del paciente con sangrado digestivo alto implica valorar el estado hemodinámico para determinar el manejo. Esta debe incluir la valoración de la vía aérea, respiración y circulación del paciente $(2,6,8)$. Mediante el examen físico se puede determinar la estabilidad hemodinámica del paciente según los siguientes signos clínicos documentados: (6) -Taquicardia en reposo: sugiere hipovolemia de leve a moderada en la cual hay pérdida sanguínea inferior a $15 \%$.

-Hipotensión ortostática: indica pérdida sanguínea mínima de $15 \%$.

-Hipotensión supina: evidencia pérdida sanguínea de al menos $40 \%$.

-Presencia de signos de abdomen agudo, como dolor abdominal severo asociado a dolor 
al rebote o defensa muscular involuntaria, incrementa la preocupación por una posible perforación, que requiere una mayor evaluación previo a la endoscopia.

En pacientes hemodinámicamente inestables se debe asegurar el acceso intravenoso mediante 2 catéteres calibre 18 de ser posible, y se debe iniciar resuscitación mediante fluidos IV inmediatamente, sin esperar hasta que el paciente sea trasladado a una unidad de cuidados intensivos. Se requiere mantener al paciente bajo vigilancia continua de su frecuencia cardiaca, presión arterial y oxigenación $(2,6,8,11)$. Se requerirá transfundir aquellos pacientes con un sangrado activo e hipovolemia, que persistan hemodinámicamente inestables, a pesar de contar con niveles de hemoglobina normal y apropiada resucitación con fluidos. Pacientes sin sangrado activo, que con adecuada resucitación con fluidos pasen a un estado hemodinámico estable, pero que sus niveles de hemoglobina sean menores a $9 \mathrm{~g} / \mathrm{dl}$ con un alto riesgo de eventos cardiovasculares o menores a $8 \mathrm{~g} / \mathrm{dl}$ con bajo riesgo de eventos cardiovasculares, también requerirán transfusiones sanguíneas con globulos rojos empacádos $(6,8)$.

Z

Posterior a la estabilización del paciente, se debe administrar medicación, iniciando por supresores de ácido, para lo cual tipicamente se utiliza inhibidores de la bomba de protones, tal como Omeprazol o Pantoprazol IV, los cuales se suelen administrar previo a la endoscopia, así mismo también se administra gastroprocinéticos, como la cisaprida o metoclopramida, para mejorar la visibilidad al momento de la endoscopia y no encontrar sangre, coágulos o restos alimenticios $(2,6,8)$.

Respecto a la úlcera péptica, durante la endoscopia, y según los hallazgos de esta, se puede utilizar la clasificación de Forrest, previamente descrita, para determinar el tratamiento que requieren. Se recomienda tratamiento endoscópico en las úlceras con sangrado activo (Forrest la y lb) y con vaso visible no sangrante (Ila); mas no se recomienda ni se justifica en las úlceras con máculas pigmentadas planas o de base limpia, en donde solo se recomienda tratamiento conservador, con el uso de terapia antisecretora, así mismo se debe iniciar terapia con inhibidores de la bomba de protones en aquellos pacientes que requirieron hemostasia mediante endoscopia (2) El tratamiento con inhibidores de bomba de protones en pacientes con úlcera péptica con lesiones de bajo riesgo, generalmente se les prescribe tratamiento por al menos 4 a 8 semanas desde el momento del diagnóstico (2, 8).

Pacientes que cursan con esofagitis diagnosticada por endoscopia raramente requieren manejo endoscópico, a menos que se evidencie estigmas de sangrado reciente. Suelen requerir la administración de inhibidores de la bomba de protones por 8 a 12 semanas, seguida de una endoscopia para descartar esófago de Barrett, caracterizado por tratarse de una metaplasia intestinal de la mucosa esofágica, misma que se considera como una lesión premaligna para carcinoma esofágico (3). En cuanto al Síndrome de Mallory-Weiss, el paciente también debe ser valorado de acuerdo con su estabilidad hemodinámica, y debe ser estabilizado previo a la endoscopia como parte de su tratamiento inicial $(8,11)$. Una vez que el paciente está estable, es seguro realizar la endoscopia para confirmar el diagnóstico y definir el tratamiento. En aquellas lesiones con sangrado activo se debe realizar terapia 
endoscópica, sin embargo, se recomienda el manejo conservador mediante supresores de ácido, como inhibidores de bomba de protones previamente mencionados, en lesiones no sangrantes. El uso de antieméticos, como metoclopramida y proclorperazina, se reserva para pacientes que persisten con náuseas y vómitos (11).

\section{COMPLICACIONES}

Desde el momento en que un paciente cursa con sangrado del tracto digestivo corre el riesgo de presentar complicaciones, principalmente de resangradoymortalidad. Esimportanteestratificar el riesgo con estudios complementarios como endoscopia, exámenes de laboratorio y clínica, enfocándose en la estabilidad hemodinámica, niveles de hemoglobina, sangrado activo, tamaño y localización de úlcera (6). Además, se podría utilizar las escalas de riesgo como Rockall y Blatchford que aportan datos de prioridad e intensidad para el manejo de estos pacientes (6).

Los riesgos durante una endoscopia alta son: broncoaspiración, reacciones adversas a medicamentos de sedación, perforación gastrointestinal, y aumento de sangrado durante el procedimiento (6). Las complicaciones principales en cualquier sangrado digestivo alto son shock hipovolémico como complicación más inmediata y anemia por deficiencia de hierro que se se manifestaría en los sangrados más crónicos $(1,10)$.

Las complicaciones de una úlcera péptica son diferentes a las de otras patologías causantes de sangrado digestivo alto (10). Se incluye resangrado, perforación de la úlcera, provocando lo que se conoce como Síndrome de perforación de viscera hueca, penetración de la úlcera y obstrucción, especialmente cuando la úlcera se encuentra ubicada en el píloro gastrico o bulbo duodenal (10).

\section{PRONÓSTICO}

El pronóstico dependerá de la patología subyacente. Por ejemplo, el síndrome de Mallory-Weiss es una patología que sana muy rápidamente y que no requiere endoscopia control para valorar la evolución de la lesió $(3,11)$. Sin embargo la mortalidad depende de si el paciente presentó sangrado activo en el momento de la endoscopia, la edad del paciente y sus comorbilidades (11). Se debe de considerar las patologías agudas y crónicas del paciente, además de la cantidad de sangrado (14). Shock, sepsis, lesión renal y cirrosis son factores que incrementan el riesgo de mortalidad en estos pacientes (14).

La recurrencia de sangrado es frecuente, se han hecho análisis que indican que factores significativos para que esto ocurra son enfermedad metastásica, egreso sin indicación médica y estancia hospitalaria mayor a 3 días (15). El hecho de ser mayor a 60 años implica una mortalidad de 12 a $25 \%$ en este grupo etario. Los pacientes con shock hemorrágico presentaron una mortalidad de hasta $30 \%$ (15). Los siguientes son factores de riesgo que incrementan mortalidad, recurrencia de sangrado, necesidad de endoscopia o cirugía: edad mayor a 60 años, comorbilidades severas, inestabilidad hemodinámica, sangrado activo, hipotensión, 6 unidades de glóbulos rojos empacados en transfusión sanguínea, tiempo de sangrado y coagulopatía severa $(2,3,15)$.

Se puede utilizar la clasificación de Forrest para 
determinar el riesgo de re-sangrado en caso de que no se haya brindado la terapia adecuada. La úlcera Forrest IA tiene un $100 \%$ de probabilidad, la IB un $55 \%$, la IIA un $43 \%$, la IIB un $22 \%$, la IIc un $10 \%$ y la III un $5 \%$ de riesgo de re-sangrado $(1,2)$.

Con respecto a las úlceras pépticas es importante la erradicación de $\mathrm{H}$. pylori y la suspensión de AINES para evitar la recurrencia de la misma y asegurar su óptima resolución (10).

\section{CONCLUSIONES}

El sangrado digestivo alto es una emergencia médica frecuente que implica alta mortalidad en personas que presentan factores de riesgo. Su etiología se divide en causas no variceales, las cualessonlasmásfrecuentes, ycausasvariceales. Los factores de riesgo conocidos se asocian mayormente a la ingesta de medicamentos nocivos así como a la infección por $\mathrm{H}$. pylori. Las manifestaciones clínicas más comunes son la hematemesis y melena. El manejo se basa en la estabilización hemodinámica como paso inicial y en definir el origen del sangrado mediante una endoscopia, la cual en ocasiones es diagnóstica y terapéutica a su vez. Su pronóstico está relacionado con la etiología subyacente y los factores de riesgo asociados así como edad del paciente, comorbilidades, entre otros.

\section{BIBLIOGRAFÍA}

1. DynaMed [Internet]. Ipswich (MA): EBSCO Information Services. 1995 - . Record No. T905671, Acute Nonvariceal Upper Gastrointestinal Bleeding; [updated 2018 Nov 30, cited San Jose, CR. $20 / 04 / 2020]$. Available from https://www.dynamed.com/topics/dmp AN T905671.

2. Brenes Rojas Y. Sangrado digestivo alto por úlcera péptica. Revista medica de Costa Rica y centroamerica. 2016; LXXIII(620):693-700.

3. Klein A, Gralnek I. Acute, nonvariceal upper gastrointestinal bleeding. Current Opinion in Critical Care. 2015;21(2):154-162.

4. Laine L. Upper Gastrointestinal Bleeding Due to a Peptic Ulcer. New England Journal of Medicine. 2016;374(24):2367-2376.

5. Rockey D. Causes of upper gastrointestinal bleeding in adults. Uptodate [Internet]. 2018 [cited 20 April 2020];. Available from: https://www.uptodate.com/contents/causes-of-upper-gastrointestinal-bleeding-in-adults

6. Saltzman J. Approach to acute upper gastrointestinal bleeding in adults. Uptodate [Internet]. 2019 [cited 20 April 2020]; . Available from: https://www.uptodate.com/contents/approach-to-acute-upper-gastrointestinal-bleeding-in-adults/contributors\#! 
7. DynaMed [Internet]. Ipswich (MA): EBSCO Information Services. 1995 - . Record No. T905063, Acute Upper Gastrointestinal Bleeding in Children - Approach to the Patient; [updated 2018 Nov 30, cited San Jose, CR. 20 /04/2020]. Available from https://www.dynamed.com/topics/ dmp AN T905063.

8. Stanley A, Laine L. Management of acute upper gastrointestinal bleeding. BMJ. 2019;364:I536.

9. Vakil N. Peptic ulcer disease: Clinical manifestations and diagnosis. Uptodate [Internet]. 2020 [cited 20 April 2020];. Available from: https://www.uptodate.com/contents/peptic-ulcer-disease-clinical-manifestations-and-diagnosis/print\#!

10. DynaMed [Internet]. Ipswich (MA): EBSCO Information Services. 1995 - . Record No. T116374, Peptic Ulcer Disease; [updated 2018 Nov 30, cited San Jose, CR. 20 /04/2020 ]. Available from https://www.dynamed.com/topics/dmp AN T116374. Registration and login required.

11. Guelrud M. Mallore-Weiss Syndrome. Uptodate [Internet]. 2019 [cited 20 April 2020];. Available from: https://www.uptodate.com/contents/mallory-weiss-syndrome/contributors

12. DynaMed [Internet]. Ipswich (MA): EBSCO Information Services. 1995 - . Record No. T113964, Esophageal Varices; [updated 2018 Nov 30, cited San Jose, CR. 20 /04/2020]. Available from https://www.dynamed.com/topics/dmp AN T113964.

13. Perencevich M, Saltzman J. Evaluation of occult gastrointestinal bleeding. Uptodate [Internet]. 2018 [cited 20 April 2020];. Available from: https://www.uptodate.com/contents/evaluation-of-occult-gastrointestinal-bleeding\#!

14. Cook D, Guyatt G. Prophylaxis against Upper Gastrointestinal Bleeding in Hospitalized Patients. New England Journal of Medicine. 2018;378(26):2506-2516.

15. Upchurch B. Upper Gastrointestinal Bleeding (UGIB): Practice Essentials, Background, Etiology [Internet]. Emedicine.medscape.com. 2019 [cited 20 April 2020]. Available from: https://emedicine.medscape.com/article/187857-overview

\section{(9) $\Theta \Theta$}

